Gut and Liver, Vol. 10, No. 1, January 2016, pp. 140-146

\title{
Effects of Surgical Methods and Tumor Location on Survival and Recurrence Patterns after Curative Resection in Patients with T2 Gallbladder Cancer
}

\author{
Woohyun Jung, Jin-Young Jang, Mee Joo Kang, Ye Rim Chang, Yong Chan Shin, Jihoon Chang, and Sun-Whe Kim \\ Department of Surgery and Cancer Research Institute, Seoul National University College of Medicine, Seoul, Korea
}

Background/Aims: Extended cholecystectomy is generally recommended for patients with $\mathrm{T} 2$ gallbladder cancer. However, few studies have assessed the extent of resection relative to T2 gallbladder tumor location. This study analyzed the effects of surgical methods and tumor location on survival outcomes and tumor recurrence in patients with T2 gallbladder cancer. Methods: Clinicopathological characteristics, extent of resection, survival rates, and recurrence patterns were retrospectively analyzed in 88 patients with pathologically confirmed T2 gallbladder cancer. Results: The 5-year disease-free survival rate was $65.0 \%$. Multivariate analysis showed that lymph node metastasis was the only independent risk factor for poor 5-year disease-free survival rate. Survival outcomes were not associated with tumor location. Survival tended to be better in patients who underwent extended cholecystectomy than in those who underwent simple cholecystectomy. Recurrence rate was not affected by surgical method or tumor location. Systemic recurrence was more frequent than local recurrence without distant recurrence. Gallbladder bed recurrence and liver recurrence were relatively rare, occurring only in patients with liver side tumors. Conclusions: Extended cholecystectomy is the most appropriate treatment for T2 gallbladder cancer. However, simple cholecystectomy with regional lymph node dissection may be appropriate for patients with serosal side tumors. (Gut Liver 2016;10:140-146)

Key Words: Gallbladder neoplasms; Cholecystectomy; Survival; Recurrence; Tumor location

\section{INTRODUCTION}

Gallbladder (GB) cancer traditionally has a dismal prognosis. ${ }^{1,2}$ The only curative therapy is surgical resection. However, many patients are diagnosed too late due to the lack of specific symptoms, and GB cancer has a propensity for early dissemination, thus preventing curative surgery. Regular health examinations and laparoscopic surgery have increased the incidental diagnosis of early GB cancer, increasing the probability of curative resection and improving survival outcomes.

The extent of resection is mainly determined by the depth of tumor invasion. T2 GB cancer is defined as a tumor invading the perimuscular connective tissue or subserosal layer but not invading the serosa. ${ }^{3}$ Extended cholecystectomy is generally recommended for patients with T2 GB cancer, a procedure that includes cholecystectomy, liver wedge resection around the GB bed and regional lymphadenectomy. ${ }^{4}$ Previous studies have reported that extended surgery improves survival rates. ${ }^{5-11}$ However, due to the rarity of GB cancer and its varied incidence in different countries, to date no well-designed randomized control study or prospective cohort study has assessed the effect of extent of surgery on outcomes in patients with T2 GB cancer. The recommendation of radical surgery for T2 GB cancer is based on low level of evidence.

Half of the GB is attached to the inferior surface of liver (liver side) and the other side faces the peritoneum (serosal side). Venous and lymphatic drainage of GB cancers differ according to the anatomic location of the tumor, especially for tumors located on the liver and serosal sides, ${ }^{12}$ thus affecting the patterns of tumor spread. ${ }^{13}$ Little is known, however, about the effects of tumor location on the outcomes of GB resection, and it remains unclear whether hepatic resection is necessary for T2 GB tumors located on the serosal side.

Therefore, this study analyzed the effects of tumor location and surgical methods on survival outcomes and recurrence patterns of T2 GB cancer after curative resection.

Correspondence to: Jin-Young Jang

Department of Surgery, Seoul National University College of Medicine, 101 Daehak-ro, Jongno-gu, Seoul 03080, Korea

Tel: +82-2-2072-2194, Fax: +82-2-741-2194, E-mail: jangjy4@snu.ac.kr

Received on February 12, 2015. Revised on April 14, 2015. Accepted on April 15, 2015. Published online September 9, 2015

pISSN 1976-2283 eISSN 2005-1212 http://dx.doi.org/10.5009/gnl15080

() This is an Open Access article distributed under the terms of the Creative Commons Attribution Non-Commercial License (http://creativecommons.org/licenses/by-nc/4.0) which permits unrestricted non-commercial use, distribution, and reproduction in any medium, provided the original work is properly cited. 


\section{MATERIALS AND METHODS}

Between April 1998 and December 2010, 144 patients with pathologically confirmed T2 stage GB cancer underwent surgery in Seoul National University Hospital. Patients who did not have preoperative computed tomography (CT) scans and pathologic slides for image and pathology review $(n=38)$, those who had distant metastasis at the time of operation $(n=13)$, and those who had GB cancer combined other malignancies ( $n=2)$, were excluded. Curative resection was defined as the absence of surgical margin involvement on pathologic reports and the absence of metastases to distant organs. Among the patients who underwent cholecystectomy without lymph node (LN) dissection, those with suspected LN metastasis on preoperative radiologic images and/or operative findings and confirmed as having metastasis by review of follow-up studies were defined as having undergone noncurative resection and excluded. Patients at high risk of dissemination, such as those with preoperative GB perforation $(n=1)$ or who underwent percutaneous GB drainage with unsuspected GB cancer $(n=2)$, were also excluded. Finally, the medical records and radiologic imaging results of 88 patients with T2 GB cancer who underwent curative resection were retrospectively reviewed. Patients' demographic and clinical characteristics, including age, gender, clinical history, preoperative radiologic image results and type of operation, were investigated. Preoperative CT images were reviewed to determine preoperative clinical stage. Tumor location was divided into two groups, such as serosal side and liver side, by preoperative CT and pathologic slide review. Serosal side tumors were defined as those with no contact point with the liver bed; all others with total or partial contact with liver were classified as liver side tumors (Fig. 1). Type of operation was categorized as simple or extended cholecystectomy. Laparoscopic cholecystectomy and open cholecystectomy without liver resection were categorized as simple cholecystectomy (SC). Cholecystectomy with hepatic wedge resection including regional LN dissection was categorized as extended cholecystectomy (EC). In our institution, cystic duct LN, common bile duct LN, hepatoduodenal ligament LN and posterior pancreatoduodenal LN were dissected routinely for regional LN dissection. Pathological staging was based on the seventh edition of the American Joint Committee on Cancer (AJCC) Manual. ${ }^{3}$ Tumors were classified grossly as papillary, nodular and sclerosing types. Adjuvant therapy was reviewed. Adjuvant chemotherapy and radiation therapy was recommended for patients with T2 GB cancer, especially for patients with LN metastasis, if the patient's general condition was tolerable. Recurrence patterns, including time to recurrence, recurrence site and disease-free survival (DFS), were also investigated.

Statistical analyses were performed using IBM SPSS version 21.0 (IBM Co., Armonk, NY, USA). Categorical variables were compared using the Pearson chi-square or the Fisher exact test and continuous variables using the Student t-test or the MannWhitney U test. Cumulative survival rates were estimated using the Kaplan-Meier method and compared using the log-rank test. Cox proportional hazards regression analysis included variables relatively associated with survival outcome on univariate analyses ( $p$-values $\leq 0.1$ ). $p$-values $\leq 0.05$ were considered statistically significant.

This study was approved by the Institutional Review Board of the Seoul National University Hospital (H-1401-063-550).

\section{RESULTS}

\section{Clinicopathological characteristics}

The clinicopathological characteristics of the patients are shown in Table 1 . The 88 patients with pathologically confirmed, curatively resected T2 GB cancer included 36 men and 52 women, with mean age of 65.0 years and median follow-up duration of 66.5 months (range, 6.4 to 190.5 months). Sixteen patients underwent simple cholecystectomy and 72 underwent extended cholecystectomy. Combined common bile duct resection was done for five patients with suspicious cystic duct resection margin involvement $(n=3)$ or presence of choledochal cyst $(n=2)$. Tumors were located on the liver side of the GB in

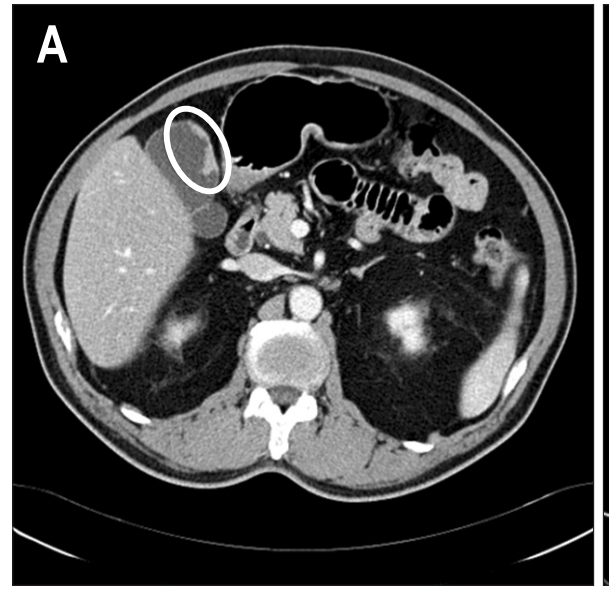

Serosal side tumor

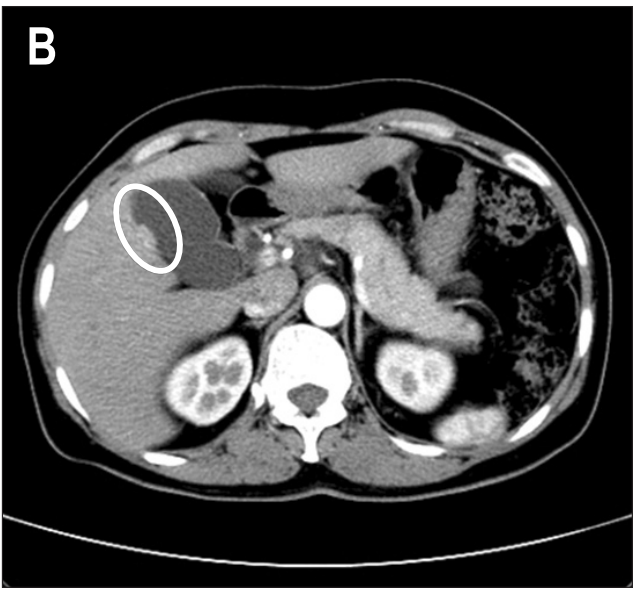

Liver side tumor
Fig. 1. Computed tomography scans of tumors on the serosal (A) and liver side (B). 
Table 1. Clinicopathological Characteristics according to Tumor Location

\begin{tabular}{|c|c|c|c|c|}
\hline Characteristic & Total $(\mathrm{n}=88)$ & Liver side $(n=62)$ & Serosal side $(n=26)$ & $\mathrm{p}$-value \\
\hline Age, yr & $65.0(39-84)$ & $65.1(39-84)$ & $63.8(43-80)$ & 0.572 \\
\hline Sex & & & & 0.484 \\
\hline Male & $36(40.9)$ & $27(43.5)$ & $9(34.6)$ & \\
\hline Female & $52(59.1)$ & $35(56.5)$ & $17(65.4)$ & \\
\hline Operation method & & & & 0.375 \\
\hline Extended cholecystectomy & $72(81.8)$ & $49(79.0)$ & $23(88.5)$ & \\
\hline Simple cholecystectomy & 16 (18.2) & $13(21.0)$ & $3(11.5)$ & \\
\hline Gross type* & & & & 0.116 \\
\hline Papillary & 41 (47.7) & $30(49.2)$ & $11(44.0)$ & \\
\hline Nodular & $33(38.4)$ & $20(32.8)$ & $13(52.0)$ & \\
\hline Sclerosing & $12(14.0)$ & $11(18.0)$ & $1(4.0)$ & \\
\hline Lymph node metastasis & & & & 0.798 \\
\hline Positive & $29(33.0)$ & $21(38.9)$ & $8(32.0)$ & \\
\hline Negative & $50(56.8)$ & $33(61.1)$ & $17(68.0)$ & \\
\hline Lymphovascular invasion & $27(30.7)$ & 19 (30.6) & $8(30.8)$ & 0.590 \\
\hline Perineural invasion & $13(14.8)$ & $11(17.7)$ & $2(7.7)$ & 0.191 \\
\hline Adjuvant chemotherapy & $29(33.0)$ & $18(29.0)$ & $11(42.3)$ & 0.320 \\
\hline Adjuvant radiotherapy & $27(30.7)$ & $15(24.2)$ & $12(46.2)$ & 0.074 \\
\hline
\end{tabular}

Data are presented as mean (range) or number (\%).

*Two cases were missing because of the lack of information about gross type on operation record and pathologic report.

A Total population

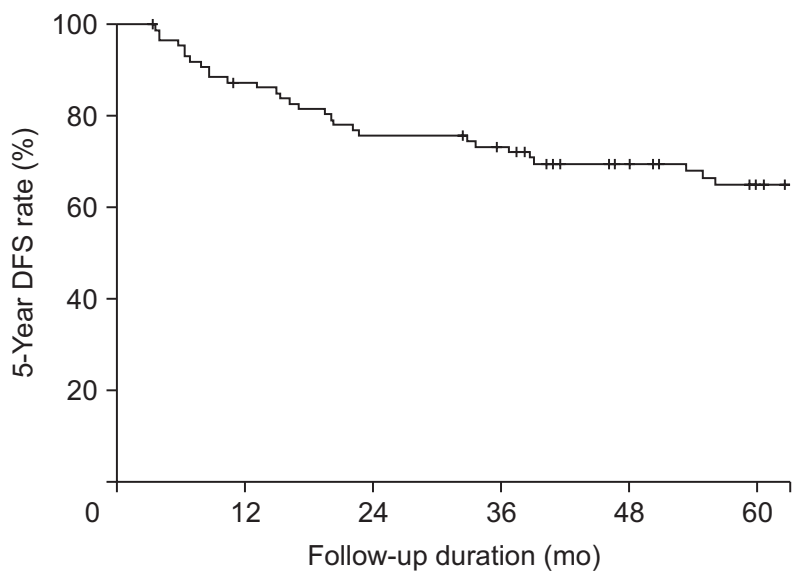

C Extended cholecystectomy

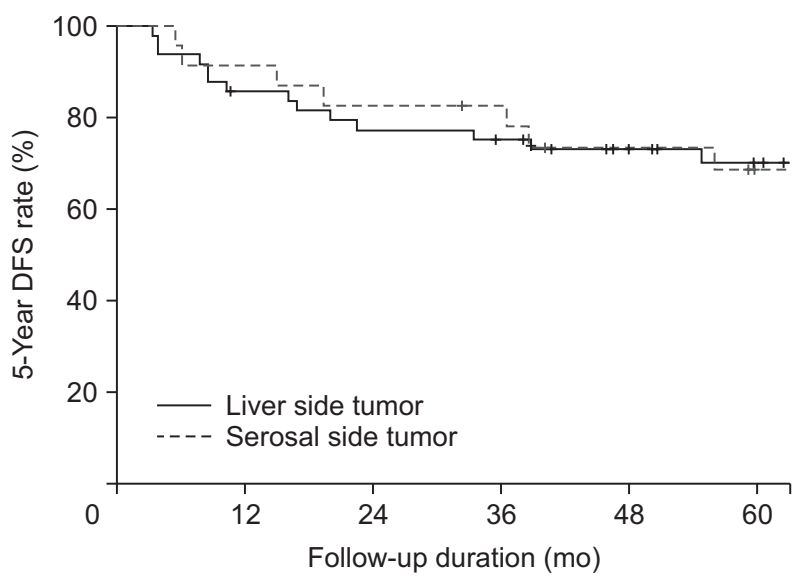

B Simple cholecystectomy

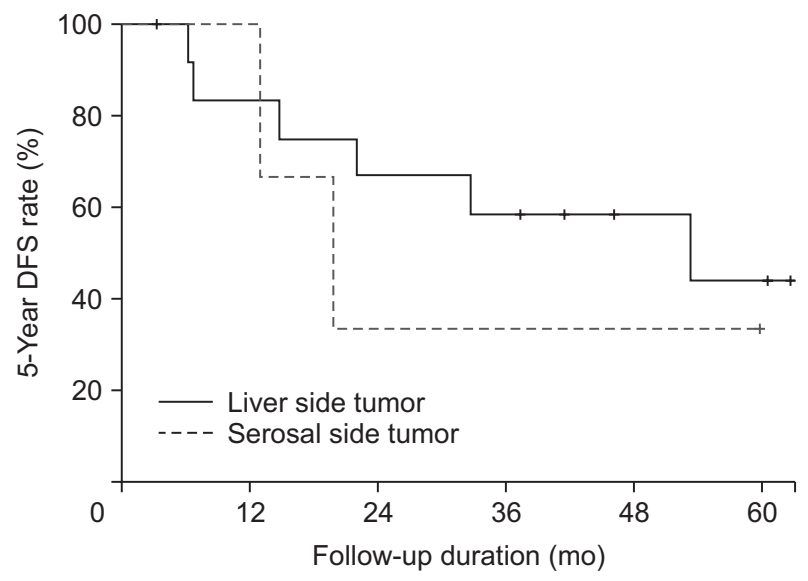

Fig. 2. Kaplan-Meier analysis of the 5-year disease-free survival (DFS) rate after curative resection in patients with $\mathrm{T} 2$ gallbladder cancer. (A) Total population. (B) Simple cholecystectomy. (C) Extended cholecystectomy. There was no significant difference in the 5-year diseasefree survival rate between patients with liver-side and serosal-side tumors who underwent simple cholecystectomy (B) (43.7\% vs 33.3\%, $\mathrm{p}=0.578)$ or extended cholecystectomy (C) (70.1\% vs 68.5\%, $\mathrm{p}=0.986)$. 
62 patients (70.5\%) and on the serosal side in 26 (29.5\%). Gross morphologic analysis showed that 41 tumors (47.7\%) were papillary type, 33 (38.4\%) were nodular type and 12 (14.0\%) were sclerosing type. The mean number of acquired lymph nodes was 7.5 of 79 patients who underwent LN dissection. LN metastases were found in 29 patients (33.0\%), and no LN metastases in 50 (56.8\%); the remaining nine patients did not undergo LN dissection, thus preventing evaluation of LN metastasis status. Among 29 patients with LN metastases, 27 patients had metastatic LN in group 1 regional LN according to seventh AJCC manual and two patients had posterior pancreatoduodenal LN metastases. Lymphovascular invasion was observed in 27 patients (30.7\%) and perineural invasion in 13 (14.8\%). Twenty-nine patients (33.0\%) received adjuvant chemotherapy, and 27 (30.7\%) re- ceived adjuvant radiotherapy. There were no significant differences in gender, age, extent of resection, rates of LN metastasis, lymphovascular invasion, perineural invasion and adjuvant therapy between patients with liver side and serosal side tumors.

\section{Survival outcome}

The 5-year overall survival (OS) rate of the 88 patients was $69.4 \%$ and their 5-year DFS rate was 65.0\% (Fig. 2A). Univariate and multivariate analyses of the associations between clinicopathological factors and 5-year DFS rate are shown in Table 2. Only LN metastasis (relative risk [RR], 2.573; 95\% confidence interval [CI], 1.103 to $6.000 ; \mathrm{p}=0.029$ ) was identified as an independent risk factor for poor 5-year DFS. Survival was relatively better in patients who underwent EC than SC, but the difference

Table 2. Risk Factors for Disease-Free Survival in Patients with T2 Gallbladder Cancer

\begin{tabular}{|c|c|c|c|c|c|}
\hline Risk factor & 5-Year DFS, \% & p-value & Relative risk & $95 \% \mathrm{CI}$ & $\mathrm{p}$-value \\
\hline Age, yr & & 0.443 & & & \\
\hline$\leq 70$ & 63.1 & & - & - & - \\
\hline$>70$ & 69.3 & & & & \\
\hline Sex & & 0.576 & & & \\
\hline Male & 61.2 & & - & - & - \\
\hline Female & 67.8 & & & & \\
\hline Tumor location & & 0.983 & & & \\
\hline Liver side & 65.2 & & - & - & - \\
\hline Serosal side & 64.5 & & & & \\
\hline Operation method* & & 0.053 & & & \\
\hline Extended cholecystectomy & 69.5 & & & & \\
\hline Simple cholecystectomy & 42.7 & & 2.526 & $0.918-6.953$ & 0.073 \\
\hline Gross type & & 0.511 & & & \\
\hline Papillary & 66.1 & & - & - & - \\
\hline Nodular & 67.5 & & & & \\
\hline Sclerosing & 57.1 & & & & \\
\hline Lymph node metastasis* & & $<0.001$ & & & \\
\hline Positive & 44.6 & & 2.573 & $1.103-6.000$ & 0.029 \\
\hline Negative & 80.2 & & & & \\
\hline Lymphovascular invasion & & 0.194 & & & \\
\hline Positive & 59.3 & & - & - & - \\
\hline Negative & 68.5 & & & & \\
\hline Perineural invasion* & & 0.031 & & & \\
\hline Positive & 46.2 & & 1.525 & $0.607-3.831$ & 0.369 \\
\hline Negative & 68.5 & & & & \\
\hline Adjuvant chemotherapy & & 0.120 & & & \\
\hline Yes & 52.4 & & & & \\
\hline No & 71.2 & & & & \\
\hline Adjuvant radiotherapy* & & 0.018 & & & \\
\hline Yes & 45.5 & & 1.798 & $0.877-4.470$ & 0.181 \\
\hline No & 73.7 & & & & \\
\hline
\end{tabular}

DFS, disease-free survival; CI, confidence interval.

*Variables included in multivariate analysis. 
was not statistically significant (RR, 2.526; 95\% CI, 0.918 to 6.953; $p=0.073$ ). Tumor location was not associated with DFS rate. Subgroup analyses according to tumor location and extent of resection showed no differences in 5-year survival rates between patients with liver side and serosal side tumors who underwent SC (43.7\% vs 33.3\%, $\mathrm{p}=0.578)$ or EC (70.1\% vs $68.5 \%$, $\mathrm{p}=0.986$ ) (Fig. 2B and C).

\section{Recurrence pattern}

The overall recurrence rate was $18.2 \%$ after a median followup of 66.5 months. The recurrence rate was similar in patients who had undergone SC or EC (25.0\% vs $16.7 \%, \mathrm{p}=0.322)$ and in patients with liver or serosal side tumors (17.7\% vs $19.2 \%$, $p=0.544)$. Recurrence patterns are summarized in Fig. 3. LN was the most frequent site of recurrence (8.5\%). Five patients experienced regional and four experienced paraaortic LN recurrences. There was no statistically significant difference in regional LN recurrence rate between the SC and EC groups (12.5\% vs 4.2\%, $\mathrm{p}=0.223$ ). Only one patient experienced regional LN recurrence without distant recurrence, with this patient undergoing EC for a serosal side tumor. Systemic recurrence was more frequent than locoregional without distant recurrence. Of the 88 patients, 14 (15.9\%) had distant recurrence with or without locoregional recurrence, whereas only two (2.3\%) had locoregional recurrence without distant recurrence. Local recurrence occurred significantly more frequently after SC than EC (25.0\% vs 5.6\%, $\mathrm{p}=0.034$ ). The four (of 16) patients with recurrences after SC all had locoregional recurrence with or without distant recurrence. Focusing on anatomic location of tumor, local (8.1\% vs $11.5 \%$, $\mathrm{p}=0.438)$ and distant (17.7\% vs $11.5 \%, \mathrm{p}=0.353)$ recurrence rates were similar in patients with liver and serosal side tumors. Recurrences in the GB bed $(n=2)$ and liver $(n=3)$ were observed only in patients with liver side tumors but they were not affected by type of surgery. There was no GB bed and liver recurrence in patients with serosal side tumors.

\section{DISCUSSION}

Various studies have reported survival outcomes in patients with T2 GB cancer after surgical resection, with 5-year OS rates reported to range from 24\% to $77.8 \% .^{7,11,14-24}$ Most of these studies, however, were retrospective in design and included about 30 to 40 patients. Although other studies were based on national registry data, their survival results were poorer than those from individual institutions. ${ }^{17,19,24}$ National registry data may have included patients who underwent noncurative resection or with inconsistent pathologic reports. Indeed, we found that the 5-year OS rate in 16 patients noncuratively resected for T2 GB cancer, including those with preoperative perforation, percutaneous GB drainage or distant metastatic lesions, was much poorer (12.5\%) than in those who underwent curative resection (69.4\%).

The optimal extent of surgical resection for T2 GB cancer has not been clearly determined, although several clinical guidelines recommend radical resection. The National Comprehensive Cancer Network Guidelines recommend that patients with $\mathrm{T} 1 \mathrm{~b}$ and more advanced GB cancer undergo cholecystectomy, en bloc hepatic resection of segments IV B and V and clearing all LNs

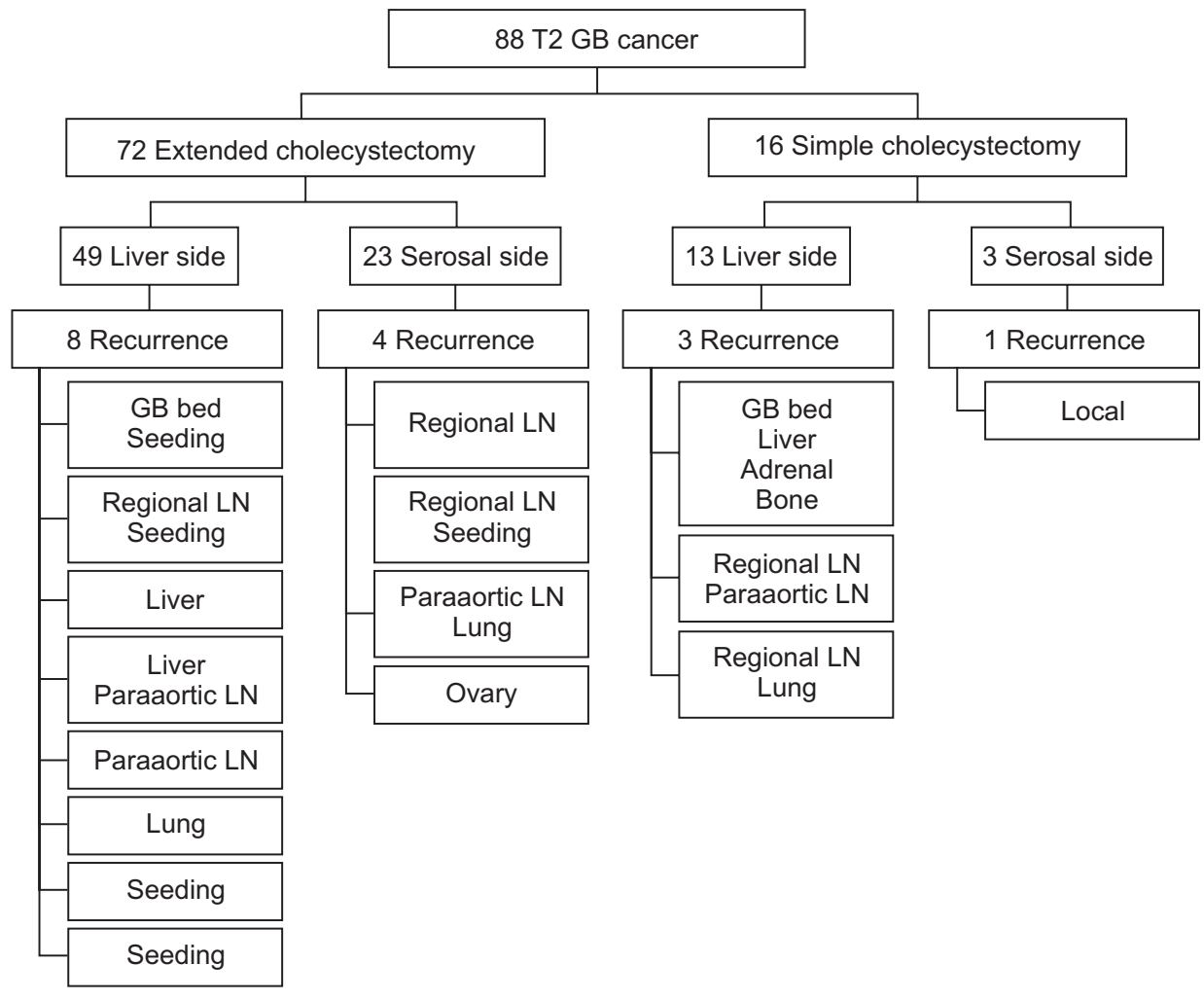

Fig. 3. Recurrence pattern after curative resection in patients with $\mathrm{T} 2$ gallbladder (GB) cancer. LN, lymph node. 
in the porta hepatis. ${ }^{4}$ Similarly, German guidelines recommend that patients with T2 and more advanced tumors undergo cholecystectomy, hepatic wedge resection around the GB bed with 3 $\mathrm{cm}$ margins or bisegmentectomy of segment IV B and regional LN dissection along the hepatoduodenal ligament. ${ }^{25}$ In Japan, open, not laparoscopic, cholecystectomy is recommended for patients with suspected GB cancer, with additional hepatectomy and lymphadenectomy recommended for patients with T2 GB cancer. ${ }^{26}$ According to the Korean guideline published in 2014, EC is generally recommended for patients with GB cancer at stage T2 or above. ${ }^{27}$ All of these recommendations, however, are based on retrospective data of small numbers of patients, with a low level of evidence.

We observed a 5-year DFS rate of $65.0 \%$ in patients curatively resected for T2 GB cancer. Multivariate analysis found that LN metastasis, observed in $31.9 \%$ of patients, was the only factor prognostic of survival. Previous studies have reported LN metastasis rates of 37\% to 50\%, with LN metastasis being prognostic of survival. ${ }^{5-11} \mathrm{LN}$ recurrence was the most common, observed in eight of 16 patients with tumor recurrence. EC tended to show a better 5-year DFS rate than SC, on both univariate (69.5\% vs 42.7\%, $\mathrm{p}=0.053$ ) and multivariate (RR, 2.526; 95\% CI, 0.918 to $6.953 ; p=0.073$ ) analyses. This result is comparable to previous studies, which reported that 5-year DFS rates were higher after EC (61\% to 90\%) than SC (19\% to 40\%). ${ }^{5,28-34}$

Assessments of tumor location showed no significant difference in clinicopathological factors, such as operation type, gross tumor type, lymphovascular invasion, perineural invasion and LN metastasis between patients with liver side and serosal side tumors. Their 5-year DFS rates were similar, and subgroup analysis showed that the extent of surgery for both liver side and serosal side tumors was not associated with survival outcomes. A recent multicenter study showed that rates of vascular invasion (51\% vs 19\%, p<0.01), neural invasion (33\% vs $8 \%$, $\mathrm{p}<0.01$ ), and LN metastasis ( $40 \%$ vs $17 \%, \mathrm{p}<0.01$ ) were significantly higher in patients with liver side than serosal side tumors. ${ }^{35}$ The 5-year OS rates were also significantly higher in patients with serosal side than liver side tumors (64.7\% vs $42.6 \%$, $\mathrm{p}=0.0006$ ), with liver side tumors independently associated with poor survival outcome (RR, 2.7; 95\% CI, 1.7 to 4.2 ; $\mathrm{p}<0.001$ ). The discrepancies between that study and the present work may be due to differences between the clinicopathologic features of liver side tumors in the two studies.

Analysis of recurrence patterns after curative resection is important in understanding disease characteristics and the effects of treatment. Although we found that recurrence rates were not affected by tumor location, GB bed recurrences were observed only in two patients with liver side tumors, and liver recurrences were observed only in three patients with liver side tumors, with neither observed in patients with serosal side tumors. In contrast, regional LN recurrences were frequently observed in patients with serosal side tumors. Similar results were obtained in the earlier multicenter study, with liver recurrences more frequent in patients with liver than serosal side tumors (23\% vs 3\%, $\mathrm{p}=0.003) .^{35}$ These findings indicate that EC including liver resection should be performed in all patients with liver side T2 GB cancers. However, SC with regional LN dissection, without liver resection around the GB bed, may be sufficient for patients with serosal side T2 GB cancer. This hypothesis could be supported by the previous study that reported laparoscopic cholecystectomy with LN dissection for early GB cancer without liver invasion could feasible option with comparable outcome. Eighteen patients with T1 $(n=8)$ or T2 $(n=10)$ GB cancer had undergone laparoscopic cholecystectomy with LN dissection and survived without recurrence after a median follow-up of 27 months. ${ }^{36}$ However, there was only limited result about this issue and in our study, only two patients with serosal side tumor underwent SC with LN dissection. It was too small a number to analyze. Subsequent studies with large cohort or prospective design about this issue will be needed to confirm the hypothesis.

This study had limitations. First, as it was based on retrospective data it may be subject to selection and information bias. Second, the small subgroup sizes and large proportion of patients lost to follow up may have altered the results.

LN metastasis was the most important prognostic factor in patients curatively resected for T2 GB cancer, with extended cholecystectomy tending to improve 5-year DFS rate. Tumor location did not affect survival outcome or recurrence patterns. However, no patient with serosal side tumors experienced GB bed or liver recurrence even after SC.

EC is the preferred surgical treatment for T2 GB cancer overall; however, the rarity of GB bed recurrence in patients with serosal side T2 GB cancer suggests that SC with regional LN dissection may be sufficient for these patients, if curative resection could be achieved.

\section{CONFLICTS OF INTEREST}

No potential conflict of interest relevant to this article was reported.

\section{REFERENCES}

1. Carriaga MT, Henson DE. Liver, gallbladder, extrahepatic bile ducts, and pancreas. Cancer 1995;75(1 Suppl):171-190.

2. Henson DE, Albores-Saavedra J, Corle D. Carcinoma of the gallbladder: histologic types, stage of disease, grade, and survival rates. Cancer 1992;70:1493-1497.

3. Edge SB; American Joint Committee on Cancer; American Cancer Society. AJCC cancer staging manual. 7th ed. New York: Springer, 2010.

4. Benson AB 3rd, Abrams TA, Ben-Josef E, et al. NCCN clinical practice guidelines in oncology: hepatobiliary cancers. J Natl Compr Canc Netw 2009;7:350-391.

5. Bartlett DL, Fong Y, Fortner JG, Brennan MF, Blumgart LH. Longterm results after resection for gallbladder cancer: implications for 
staging and management. Ann Surg 1996;224:639-646.

6. Kai M, Chijiiwa K, Ohuchida J, Nagano M, Hiyoshi M, Kondo K. A curative resection improves the postoperative survival rate even in patients with advanced gallbladder carcinoma. J Gastrointest Surg 2007;11:1025-1032.

7. Chijiiwa K, Nakano K, Ueda J, et al. Surgical treatment of patients with T2 gallbladder carcinoma invading the subserosal layer. J Am Coll Surg 2001;192:600-607.

8. Shimada H, Endo I, Fujii Y, et al. Appraisal of surgical resection of gallbladder cancer with special reference to lymph node dissection. Langenbecks Arch Surg 2000;385:509-514.

9. Wakai T, Shirai Y, Yokoyama N, Ajioka Y, Watanabe H, Hatakeyama K. Depth of subserosal invasion predicts long-term survival after resection in patients with T2 gallbladder carcinoma. Ann Surg Oncol 2003;10:447-454.

10. Kokudo N, Makuuchi M, Natori T, et al. Strategies for surgical treatment of gallbladder carcinoma based on information available before resection. Arch Surg 2003;138:741-750.

11. Choi SB, Han HJ, Kim CY, et al. Surgical outcomes and prognostic factors for T2 gallbladder cancer following surgical resection. J Gastrointest Surg 2010;14:668-678.

12. Fahim RB, McDonald JR, Richards JC, Ferris DO. Carcinoma of the gallbladder: a study of its modes of spread. Ann Surg 1962; 156:114-124.

13. Endo I, Shimada H, Takimoto A, et al. Microscopic liver metastasis: prognostic factor for patients with pT2 gallbladder carcinoma. World J Surg 2004;28:692-696.

14. Sasaki R, Uesugi N, Itabashi H, et al. Clinicopathological study of depth of subserosal invasion in patients with pT2 gallbladder carcinoma. J Surg Oncol 2005;92:83-88.

15. Kang CM, Lee WJ, Choi GH, et al. Does "clinical” Ro have validity in the choice of simple cholecystectomy for gallbladder carcinoma? J Gastrointest Surg 2007;11:1309-1316.

16. Shirai Y, Sakata J, Wakai T, Ohashi T, Hatakeyama K. "Extended" radical cholecystectomy for gallbladder cancer: long-term outcomes, indications and limitations. World J Gastroenterol 2012;18: 4736-4743.

17. de Aretxabala X, Roa I, Burgos L, et al. Gallbladder cancer: an analysis of a series of 139 patients with invasion restricted to the subserosal layer. J Gastrointest Surg 2006;10:186-192.

18. Foster JM, Hoshi H, Gibbs JF, et al. Gallbladder cancer: defining the indications for primary radical resection and radical reresection. Ann Surg Oncol 2007;14:833-840.

19. Wright BE, Lee CC, Iddings DM, Kavanagh M, Bilchik AJ. Management of $\mathrm{T} 2$ gallbladder cancer: are practice patterns consistent with national recommendations? Am J Surg 2007;194:820-825.

20. Mayo SC, Shore AD, Nathan H, et al. National trends in the management and survival of surgically managed gallbladder adenocarcinoma over 15 years: a population-based analysis. J Gastrointest Surg 2010;14:1578-1591.

21. Wakai T, Shirai Y, Hatakeyama K. Radical second resection pro- vides survival benefit for patients with $\mathrm{T} 2$ gallbladder carcinoma first discovered after laparoscopic cholecystectomy. World J Surg 2002;26:867-871.

22. Kim DH, Kim SH, Choi GH, et al. Role of cholecystectomy and lymph node dissection in patients with $\mathrm{T} 2$ gallbladder cancer. World J Surg 2013;37:2635-2640.

23. Goetze TO, Paolucci V. The prognostic impact of positive lymph nodes in stages T1 to T3 incidental gallbladder carcinoma: results of the German Registry. Surg Endosc 2012;26:1382-1389.

24. Goetze T0, Paolucci V. Benefits of reoperation of T2 and more advanced incidental gallbladder carcinoma: analysis of the German registry. Ann Surg 2008;247:104-108.

25. Lammert F, Neubrand MW, Bittner R, et al. S3-guidelines for diagnosis and treatment of gallstones. German Society for Digestive and Metabolic Diseases and German Society for Surgery of the Alimentary Tract. Z Gastroenterol 2007;45:971-1001.

26. Kondo S, Takada T, Miyazaki M, et al. Guidelines for the management of biliary tract and ampullary carcinomas: surgical treatment. J Hepatobiliary Pancreat Surg 2008;15:41-54.

27. Lee SE, Kim KS, Kim WB, et al. Practical guidelines for the surgical treatment of gallbladder cancer. J Korean Med Sci 2014;29:13331340.

28. Shirai Y, Yoshida K, Tsukada K, Muto T, Watanabe H. Radical surgery for gallbladder carcinoma: long-term results. Ann Surg 1992;216:565-568.

29. Yamaguchi K, Tsuneyoshi M. Subclinical gallbladder carcinoma. Am J Surg 1992;163:382-386.

30. Matsumoto Y, Fujii H, Aoyama H, Yamamoto M, Sugahara K, Suda K. Surgical treatment of primary carcinoma of the gallbladder based on the histologic analysis of 48 surgical specimens. Am J Surg 1992;163:239-245.

31. de Aretxabala XA, Roa IS, Burgos LA, Araya JC, Villaseca MA, Silva JA. Curative resection in potentially resectable tumours of the gallbladder. Eur J Surg 1997;163:419-426.

32. Oertli D, Herzog U, Tondelli P. Primary carcinoma of the gallbladder: operative experience during a 16 year period. Eur J Surg 1993;159:415-420.

33. Shirai Y, Yoshida K, Tsukada K, Muto T. Inapparent carcinoma of the gallbladder: an appraisal of a radical second operation after simple cholecystectomy. Ann Surg 1992;215:326-331.

34. Fong Y, Jarnagin W, Blumgart LH. Gallbladder cancer: comparison of patients presenting initially for definitive operation with those presenting after prior noncurative intervention. Ann Surg 2000;232:557-569.

35. Shindoh J, de Aretxabala X, Aloia TA, et al. Tumor location is a strong predictor of tumor progression and survival in T2 gallbladder cancer: an international multicenter study. Ann Surg 2015; 261:733-739.

36. Cho JY, Han HS, Yoon YS, Ahn KS, Kim YH, Lee KH. Laparoscopic approach for suspected early-stage gallbladder carcinoma. Arch Surg 2010;145:128-133. 